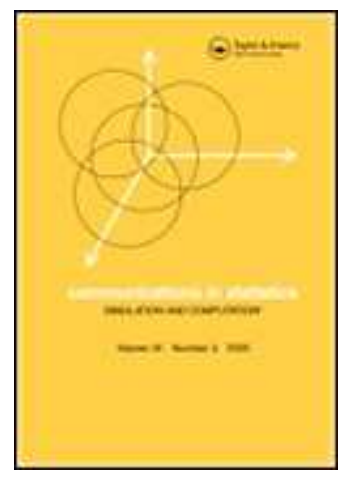

\title{
The shortest Clopper--Pearson confidence interval for binomial probability
}

\begin{tabular}{|c|c|}
\hline Journal: & Communications in Statistics - Simulation and Computation \\
\hline Manuscript ID: & LSSP-2009-0041.R2 \\
\hline Manuscript Type: & Review Article \\
\hline $\begin{array}{l}\text { Date Submitted by the } \\
\text { Author: }\end{array}$ & 23-Sep-2009 \\
\hline Complete List of Authors: & $\begin{array}{l}\text { Zielinski, Wojciech; Warsaw Unversity of Life Sciences, } \\
\text { Econometrics and Statistics }\end{array}$ \\
\hline Keywords: & $\begin{array}{l}\text { binomial proportion, confidence interval, shortest confidence } \\
\text { interval }\end{array}$ \\
\hline Abstract: & $\begin{array}{l}\text { In the paper there is shown the existence of the shortest confidence } \\
\text { interval for binomial probability. The method of obtaining such } \\
\text { interval is presented as well. }\end{array}$ \\
\hline \multicolumn{2}{|c|}{$\begin{array}{l}\text { Note: The following files were submitted by the author for peer review, but cannot be converted } \\
\text { to PDF. You must view these files (e.g. movies) online. }\end{array}$} \\
\hline Zielinski_v3.tex & \\
\hline
\end{tabular}

\section{S) ScholarONE" \\ Manuscript Central}


The shortest Clopper-Pearson confidence interval for binomial probability

\author{
Wojciech Zieliński \\ Department of Econometrics and Statistics \\ Warsaw University of Life Sciences \\ Nowoursynowska 159, 02-776 Warszawa, Poland \\ e-mail: wojtek.zielinski@statystyka.info
}

Summary. The existence of the shortest confidence interval for binomial probability is shown. The method of obtaining such an interval is presented as well.

Key words: binomial proportion, confidence interval, shortest confidence intervals

AMS 2000 Subject Classification: $62 \mathrm{~F} 25$

Consider the binomial statistical model

$$
(\{0,1, \ldots, n\},\{\operatorname{Bin}(n, \pi), 0<\pi<1\}),
$$

where $\operatorname{Bin}(n, \pi)$ denotes the binomial distribution with pdf

$$
\left(\begin{array}{l}
n \\
x
\end{array}\right) \pi^{x}(1-\pi)^{n-x}, \quad x=0,1, \ldots, n
$$

Let $X$ denote a binomial $\operatorname{Bin}(n, \pi)$ random variable. A confidence interval for the probability $\pi$ at the confidence level $\gamma$ is of the form (Clopper and Pearson, 1934)

$$
\left(F^{-1}\left(X, n-X+1 ; \gamma_{1}\right) ; F^{-1}\left(X+1, n-X ; \gamma_{2}\right)\right),
$$

where $\gamma_{1}, \gamma_{2} \in(0,1)$ are such that $\gamma_{2}-\gamma_{1}=\gamma$ and $F^{-1}(a, b ; \alpha)$ is the $\alpha$ quantile of the beta distribution with parameters $(a, b)$, i.e

$$
P_{\pi}\left\{\pi \in\left(F^{-1}\left(X, n-X+1 ; \gamma_{1}\right) ; F^{-1}\left(X+1, n-X ; \gamma_{2}\right)\right)\right\} \geq \gamma, \quad \forall \pi \in(0,1) .
$$

For $X=0$ the left end is taken to be 0 , and for $X=n$ the right end is is taken to be 1 .

Clopper and Pearson (1934) in their construction used $\gamma_{1}=(1-\gamma) / 2$ (see also Brown et al. (2001)), i.e. they applied the rule of symmetric division of $1-\gamma$ to both sides of the interval. The length of the confidence interval was not considered as a criterion. It is of interest to find the shortest confidence interval. So we want to find $\gamma_{1}$ and $\gamma_{2}$ such that the confidence interval is the shortest possible.

The problem of shortest confidence intervals was seldom considered in the past (Crow 1956, Blyth and Hutchinson 1960, Blyth and Still 1983, Casella 1986). Unfortunately, the solutions proposed are either complicated or randomization is applied. In what follows, a much simpler solution of the problem is presented. Consider the length of the confidence interval when $X=x$ is observed,

$$
d\left(\gamma_{1}, x\right)=F^{-1}\left(x+1, n-x ; \gamma+\gamma_{1}\right)-F^{-1}\left(x, n-x+1 ; \gamma_{1}\right) .
$$

Let $x$ be given. We want to find $0<\gamma_{1}<1-\gamma$ such that $d\left(\gamma_{1}, x\right)$ is minimal. The derivative of $d\left(\gamma_{1}, x\right)$ with respect to $\gamma_{1}$ equals (in what follows $B(\cdot, \cdot)$ denotes the beta function)

$$
\begin{aligned}
\frac{\partial d\left(\gamma_{1}, x\right)}{\partial \gamma_{1}}= & B(x+1, n-x)\left(1-F^{-1}\left(x+1, n-x ; \gamma+\gamma_{1}\right)\right)^{1+x-n} F^{-1}\left(x+1, n-x ; \gamma+\gamma_{1}\right)^{-x} \\
& -B(x, n-x+1)\left(1-F^{-1}\left(x, n-x+1 ; \gamma_{1}\right)\right)^{x-n} F^{-1}\left(x, n-x+1 ; \gamma_{1}\right)^{1-x} .
\end{aligned}
$$


Let

$$
\begin{aligned}
& \operatorname{LHS}\left(\gamma_{1}, x\right)=\frac{\left[1-F^{-1}\left(x+1, n-x ; \gamma+\gamma_{1}\right)\right]^{n-x-1} F^{-1}\left(x+1, n-x ; \gamma+\gamma_{1}\right)^{x}}{B(x+1, n-x)}, \\
& \operatorname{RHS}\left(\gamma_{1}, x\right)=\frac{\left[1-F^{-1}\left(x, n-x+1 ; \gamma_{1}\right)\right]^{n-x} F^{-1}\left(x, n-x+1 ; \gamma_{1}\right)^{x-1}}{B(x, n-x+1)} .
\end{aligned}
$$

Then

$$
\frac{\partial d\left(\gamma_{1}, x\right)}{\partial \gamma_{1}}=\frac{1}{\operatorname{LHS}\left(\gamma_{1}, x\right)}-\frac{1}{R H S\left(\gamma_{1}, x\right)}
$$

Because

$$
F^{-1}(x, n-x+1 ; 0)=0 \quad \text { and } \quad F^{-1}(x+1, n-x ; 1)=1,
$$

for $1<x<n-1$ we have

$$
\begin{aligned}
& \text { if } \gamma_{1} \rightarrow 0 \text { then } \operatorname{LHS}\left(\gamma_{1}, x\right)>0 \text { and } \operatorname{RHS}\left(\gamma_{1}, x\right) \rightarrow 0^{+}, \\
& \text {if } \gamma_{1} \rightarrow 1-\gamma \text { then } \operatorname{LHS}\left(\gamma_{1}, x\right) \rightarrow 0^{+} \text {and } \operatorname{RHS}\left(\gamma_{1}, x\right)>0 .
\end{aligned}
$$

Therefore, the equation

$$
\frac{\partial d\left(\gamma_{1}, x\right)}{\partial \gamma_{1}}=0
$$

has a solution.

It is easy to see that $\operatorname{LHS}(\cdot, x)$ and $R H S(\cdot, x)$ are unimodal and concave on the interval $(0,1-\gamma)$. Hence, the solution of $(*)$ is unique. Let $\gamma_{1}^{*}$ denote the solution. Because $\frac{\partial d\left(\gamma_{1}, x\right)}{\partial \gamma_{1}}<0$ for $\gamma_{1}<\gamma_{1}^{*}$ and $\frac{\partial d\left(\gamma_{1}, x\right)}{\partial \gamma_{1}}>0$ for $\gamma_{1}>\gamma_{1}^{*}$, we have $d\left(\gamma_{1}^{*}, x\right)=\inf \left\{d\left(\gamma_{1}, x\right): 0<\gamma_{1}<1-\gamma\right\}$.

It is interesting to note that for even $n$ and $x=n / 2$ the only solution of $(*)$ is $\gamma_{1}^{*}=(1-\gamma) / 2$, i.e. the symmetric confidence interval is the shortest one. Indeed, in that case

$$
\begin{aligned}
& \operatorname{LHS}\left(\gamma_{1}, x\right)=\frac{\left[1-F^{-1}\left(x+1, x ; \gamma+\gamma_{1}\right)\right]^{n-x-1} F^{-1}\left(x+1, x ; \gamma+\gamma_{1}\right)^{x}}{B(x+1, x)}, \\
& \operatorname{RHS}\left(\gamma_{1}, x\right)=\frac{\left[1-F^{-1}\left(x, x+1 ; \gamma_{1}\right)\right]^{n-x} F^{-1}\left(x, x+1 ; \gamma_{1}\right)^{x-1}}{B(x, x+1)} .
\end{aligned}
$$

Making use of the known equality

$$
F^{-1}(a, b ; q)=1-F^{-1}(b, a ; 1-q),
$$

we can rewrite equation $(*)$ in the form

$$
\left[\frac{F^{-1}\left(x, x+1 ; \gamma_{1}\right)}{F^{-1}\left(x, x+1 ; 1-\gamma-\gamma_{1}\right)}\right]^{x-1}=\left[\frac{F^{-1}\left(x+1, x ; \gamma+\gamma_{1}\right)}{F^{-1}\left(x+1, x ; 1-\gamma_{1}\right)}\right]^{x} .
$$

It is not difficult to check that the solution of the last equation is $\gamma_{1}^{*}=(1-\gamma) / 2$.

For $x=1$ we have

$$
\begin{aligned}
& \operatorname{LHS}\left(\gamma_{1}, 1\right)=\frac{\left[1-F^{-1}\left(2, n-1 ; \gamma+\gamma_{1}\right)\right]^{n-2} F^{-1}\left(2, n-1 ; \gamma+\gamma_{1}\right)}{B(2, n-1)} \\
& \operatorname{RHS}\left(\gamma_{1}, 1\right)=\frac{\left[1-F^{-1}\left(1, n ; \gamma_{1}\right)\right]^{n-1}}{B(1, n)}=n\left(1-\gamma_{1}\right)^{\frac{n-1}{n}}
\end{aligned}
$$

It is seen that

$$
\begin{aligned}
& \text { if } \gamma_{1} \rightarrow 0 \text { then } \operatorname{LHS}\left(\gamma_{1}, 1\right) \rightarrow \frac{\left[1-F^{-1}(2, n-1 ; \gamma)\right]^{n-2} F^{-1}(2, n-1 ; \gamma)}{B(2, n-1)} \text { and } \operatorname{RH} S\left(\gamma_{1}, x\right) \rightarrow n, \\
& \text { if } \gamma_{1} \rightarrow 1-\gamma \text { then } \operatorname{LHS}\left(\gamma_{1}, x\right) \rightarrow 0 \text { and } \operatorname{RH} S\left(\gamma_{1}, x\right) \rightarrow n \gamma^{\frac{n-1}{n}} .
\end{aligned}
$$


and

$$
\operatorname{RHS}\left(\gamma_{1}, 1\right)>\operatorname{LHS}\left(\gamma_{1}, 1\right) \text { for } 0<\gamma_{1}<1-\gamma \text {. }
$$

So $\frac{\partial d\left(\gamma_{1}, 1\right)}{\partial \gamma_{1}}>0$ and hence $d\left(\gamma_{1}, 1\right)$ achieves its minimal value for $\gamma_{1}=0$. For $x=1$ the shortest confidence interval is the one-sided one. Similarly, for $x=n-1$ the length $d\left(\gamma_{1}, n-1\right)$ achieves its minimal value for $\gamma_{1}=1-\gamma$ and the shortest confidence interval is also one-sided.

The value of $\gamma_{1}^{*}$ for given $\gamma, n$ and $x$ may be found numerically. Tables 1-3 give those values for $\gamma=0.95$. Clopper-Pearson confidence intervals are also shown.

Note that if $x>n / 2$, then

$$
\begin{aligned}
& \gamma_{1}^{*}(x)=(1-\gamma)-\gamma_{1}^{*}(n-x), \\
& \text { left }_{\text {short }}(x)=1-\text { right }_{\text {short }}(n-x), \\
& \text { right }_{\text {short }}(x)=1-\text { left }_{\text {short }}(n-x), \\
& \text { left }_{\text {sym }}(x)=1-\operatorname{right}_{\text {sym }}(n-x), \\
& \operatorname{right}_{\text {sym }}(x)=1-\operatorname{left}_{\text {sym }}(n-x) .
\end{aligned}
$$

\begin{tabular}{|c|c|c|c|c|c|c|c|}
\hline \multirow[b]{2}{*}{$x$} & \multicolumn{4}{|c|}{ Shortest c.i. } & \multicolumn{3}{|c|}{ Clopper-Pearson c.i. } \\
\hline & $\gamma_{1}^{*}$ & left $t_{\text {short }}$ & right $_{\text {short }}$ & length $_{\text {short }}$ & left $t_{\text {sym }}$ & right $_{\text {sym }}$ & length $_{\text {sym }}$ \\
\hline 0 & 0.00000 & 0.00000 & 0.13911 & 0.13911 & 0.00000 & 0.16843 & 0.16843 \\
\hline 1 & 0.00000 & 0.00000 & 0.21611 & 0.21611 & 0.00127 & 0.24873 & 0.24747 \\
\hline 2 & 0.00125 & 0.00261 & 0.28393 & 0.28132 & 0.01235 & 0.31698 & 0.30463 \\
\hline 3 & 0.00561 & 0.01839 & 0.34998 & 0.33159 & 0.03207 & 0.37893 & 0.34686 \\
\hline 4 & 0.00966 & 0.04318 & 0.41249 & 0.36931 & 0.05733 & 0.43661 & 0.37928 \\
\hline 5 & 0.01302 & 0.07344 & 0.47156 & 0.39812 & 0.08657 & 0.49105 & 0.40447 \\
\hline 6 & 0.01587 & 0.10763 & 0.52766 & 0.42004 & 0.11893 & 0.54279 & 0.42386 \\
\hline 7 & 0.01840 & 0.14496 & 0.58118 & 0.43622 & 0.15391 & 0.59219 & 0.43828 \\
\hline 8 & 0.02071 & 0.18496 & 0.63234 & 0.44738 & 0.19119 & 0.63946 & 0.44827 \\
\hline 9 & 0.02288 & 0.22733 & 0.68126 & 0.45393 & 0.23058 & 0.68472 & 0.45414 \\
\hline 10 & 0.02500 & 0.27196 & 0.72804 & 0.45609 & 0.27196 & 0.72804 & 0.45609 \\
\hline
\end{tabular}

Table 1. $n=20$

Table 2. $n=100$

\begin{tabular}{c||c|c|c|c||c|c|c}
\multicolumn{1}{l||}{} & \multicolumn{9}{c||}{ Shortest c.i. } & \multicolumn{3}{c}{ Clopper-Pearson c.i. } \\
$x$ & $\gamma_{1}^{*}$ & left $_{\text {short }}$ & right $_{\text {short }}$ & lengt $_{\text {short }}$ & left $_{\text {sym }}$ & right $_{\text {sym }}$ & lengt $_{\text {sym }}$ \\
\hline 5 & 0.00823 & 0.01236 & 0.10507 & 0.09272 & 0.01643 & 0.11284 & 0.09640 \\
10 & 0.01399 & 0.04462 & 0.16978 & 0.12516 & 0.04901 & 0.17622 & 0.12722 \\
15 & 0.01690 & 0.08234 & 0.22989 & 0.14755 & 0.08645 & 0.23531 & 0.14885 \\
20 & 0.01880 & 0.12299 & 0.28733 & 0.16434 & 0.12666 & 0.29184 & 0.16519 \\
25 & 0.02023 & 0.16564 & 0.34287 & 0.17723 & 0.16878 & 0.34655 & 0.17777 \\
30 & 0.02140 & 0.20984 & 0.39692 & 0.18708 & 0.21241 & 0.39982 & 0.18741 \\
35 & 0.02241 & 0.25534 & 0.44972 & 0.19438 & 0.25729 & 0.45185 & 0.19456 \\
40 & 0.02332 & 0.30197 & 0.50139 & 0.19942 & 0.30330 & 0.50279 & 0.19950 \\
45 & 0.02417 & 0.34965 & 0.55203 & 0.20238 & 0.35032 & 0.55272 & 0.20240 \\
50 & 0.02500 & 0.39832 & 0.60168 & 0.20336 & 0.39832 & 0.60168 & 0.20336
\end{tabular}


Table 3. $n=1000$

\begin{tabular}{c||c|c|c|c||c|c|c}
\multicolumn{1}{l||}{} & \multicolumn{9}{c||}{ Shortest c.i. } & \multicolumn{3}{c}{ Clopper-Pearson c.i. } \\
$x$ & $\gamma_{1}^{*}$ & left $_{\text {short }}$ & right $_{\text {short }}$ & lengt $_{\text {short }}$ & left $_{\text {sym }}$ & right $_{\text {sym }}$ & lengt $_{\text {sym }}$ \\
\hline 50 & 0.01979 & 0.03678 & 0.06473 & 0.02795 & 0.03734 & 0.06539 & 0.02806 \\
100 & 0.02165 & 0.08159 & 0.11972 & 0.03812 & 0.08211 & 0.12029 & 0.03818 \\
150 & 0.02254 & 0.12797 & 0.17317 & 0.04519 & 0.12843 & 0.17366 & 0.04523 \\
200 & 0.02312 & 0.17523 & 0.22574 & 0.05051 & 0.17562 & 0.22616 & 0.05054 \\
250 & 0.02356 & 0.22310 & 0.27771 & 0.05460 & 0.22343 & 0.27805 & 0.05462 \\
300 & 0.02391 & 0.27146 & 0.32919 & 0.05773 & 0.27172 & 0.32946 & 0.05774 \\
350 & 0.02421 & 0.32022 & 0.38027 & 0.06005 & 0.32042 & 0.38047 & 0.06005 \\
400 & 0.02449 & 0.36934 & 0.43099 & 0.06165 & 0.36947 & 0.43112 & 0.06165 \\
450 & 0.02475 & 0.41879 & 0.48138 & 0.06259 & 0.41885 & 0.48144 & 0.06259 \\
500 & 0.02500 & 0.46855 & 0.53145 & 0.06290 & 0.46855 & 0.53145 & 0.06290
\end{tabular}

Below we give a short Mathematica program for calculating $\gamma_{1}^{*}$ and the ends of the shortest confidence interval. Of course, one can also use other mathematical or statistical packages (in a similar way) to find the values of $\gamma_{1}^{*}$.

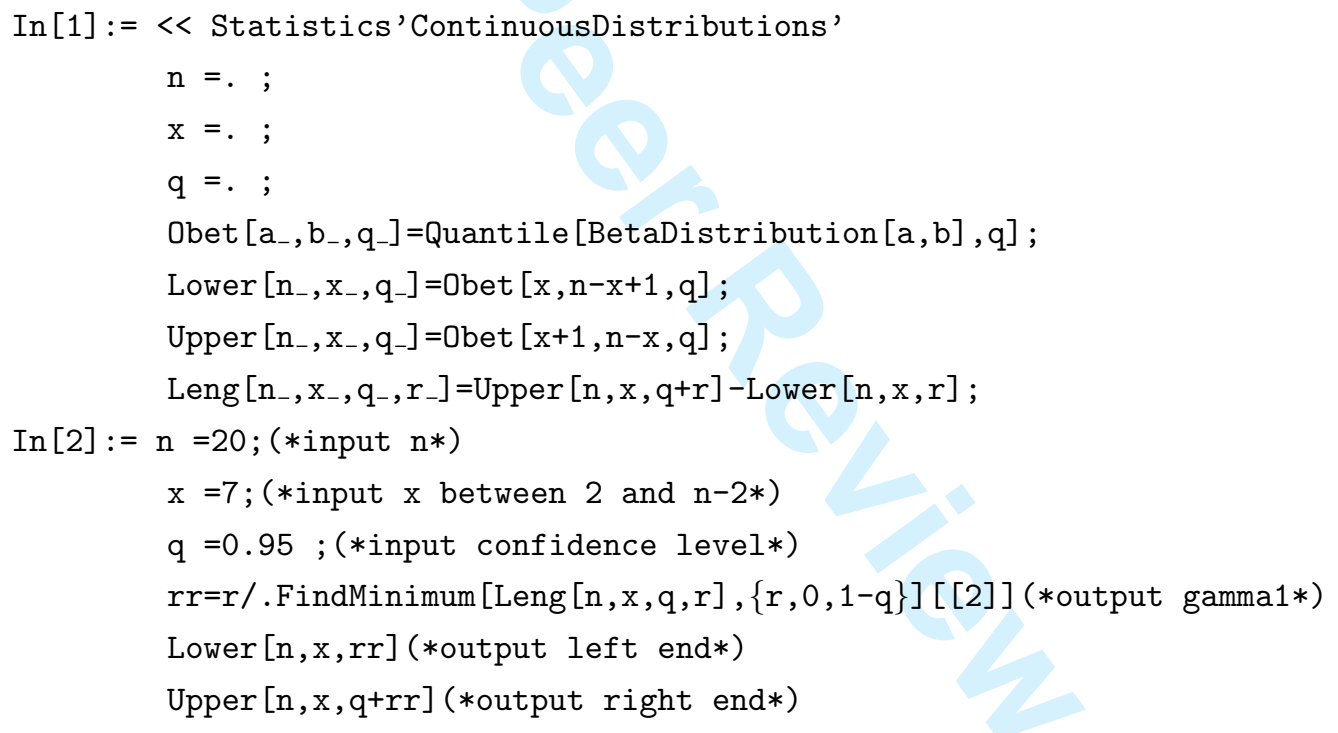

In Clopper and Pearson's times calculating quantiles of a beta distribution was numerically complicated. Nowadays, it is very easy with the aid of computer software, so using the shortest confidence interval is recommended, especially for small sample sizes $n$. 


\section{References}

Brown L. D., Cai T. T., DasGupta A. (2001) Interval Estimation for Binomial Proportion, Statistical Science, $16,101-133$

Blyth C. R., Hutchinson D. W. (1960) Table of Neyman-Shortest Unbiased Confidence Intervals for the Binomial Parameter, Biometrika 47, 381-391

Blyth C. R., Still H. A. (1983) Binomial Confidence Intervals, Journal of the American Statistical Association $78,108-116$

Casella G. (1986) Refining Binomial Confidence Intervals, The Canadian Journal of Statistics 14, 113-129

Clopper C. J., Pearson E. S. (1934), The Use of Confidence or Fiducial Limits Illustrated in the Case of the Binomial, Biometrika 26, 404-413

Crow J. L. (1956) Confidence Intervals for a Proportion, Biometrika 45, 423-435 